Journal of Applied AnALysis

Vol. 11, No. 1 (2005), pp. 95-112

\title{
SOLUTIONS OF NONLINEAR SINGULAR BOUNDARY VALUE PROBLEMS
}

\author{
H. SEBAI
}

Received September 23, 2002 and, in revised form, April 15, 2003

\begin{abstract}
We study the existence of solutions to a class of problems

$$
u^{\prime \prime}+f(t, u)=0, \quad u(0)=u(1)=0,
$$

where $f(t, \cdot)$ is allowed to be singular at $t=0, t=1$.
\end{abstract}

\section{Introduction}

Consider the singular boundary value problem (BVP)

$$
\begin{aligned}
& u^{\prime \prime}+f(t, u)=0, \\
& u(0)=u(1)=0,
\end{aligned}
$$

where $f:(0,1) \times \mathbb{R}^{k} \rightarrow \mathbb{R}^{k}, k \in \mathbb{N}$, is singular both at the end points $t=0, t=1$ and $f$ will be either a Carathéodory function, or a continuous function. A model example for a continuous function $f$ is

$$
f(t, u)=\frac{g(u)}{t^{\gamma}(1-t)^{\gamma}},
$$

2000 Mathematics Subject Classification. 34B16, 34B18.

Key words and phrases. Nonlinear ordinary differential equation, boundary value problem, singular, positive solution, superlinear method.

ISSN 1425-6908（C) Heldermann Verlag. 
where $g$ is continuous. In the case $\gamma<2$ the Dirichlet problem (1.1)-(1.2) was investigated by several authors $([1,2,3,4,5,6,7,8,9,10,11,12$, $13,14,15])$. By a solution $u$ of $(1.1)-(1.2)$ in the continuous case we mean a function $u \in C\left([0,1], \mathbb{R}^{k}\right) \cap C^{2}\left((0,1), \mathbb{R}^{k}\right)$ satisfying (1.1) everywhere and (1.2). In the Carathéodory case, this means a function $u \in C\left([0,1], \mathbb{R}^{k}\right)$ with absolutely continuous derivative $u$ which satisfies (1.1) almost everywhere and (1.2). In the case $\gamma \geq 2$ the standard method of finding a fixed point to an appropriate integral operator (see below) will not be acting in the space $C$ of all continuous functions $u:[0,1] \rightarrow \mathbb{R}^{k}$.

\section{The general framework}

The present work shows that having $\gamma \geq 2$, we shall obtain the existence of solutions for the problem (1.1)-(1.2). The results here are different. Our method of proof is based on the definition of the following subspace

$$
X_{\alpha}:=\left\{u \in C\left([0,1], \mathbb{R}^{k}\right): \sup _{t \in(0,1)} \frac{|u(t)|}{(t(1-t))^{\alpha}}<\infty\right\},
$$

$\alpha \in(0,1)$ with the norm

$$
\|u\|_{\alpha}=\sup _{t \in(0,1)} \frac{|u(t)|}{t^{\alpha}(1-t)^{\alpha}}
$$

where $C\left([0,1], \mathbb{R}^{k}\right)$ is the space of all continuous functions from $[0,1]$ into $\mathbb{R}^{k}$, and $|\cdot|$ means the Euclidean norm in $\mathbb{R}^{k}$.

Functions from $X_{\alpha}$ vanish immediately at the ends of the interval $[0,1]$ and the family of $X_{\alpha}$ is extending (increasing) when $\alpha$ decreases to 0 . For $\alpha \geq 1$, the space is not sufficiently large. It is obvious that the convergence in $X_{\alpha}$ is the uniform convergence after multiplication by the function

$$
t \rightarrow(t(1-t))^{-\alpha}
$$

and that the compactness criterion in $X_{\alpha}$ is the classical Ascoli-Arzèla's theorem after the same operation. The first result Theorem 2.1 shows conditions which guarantee that the Hammerstein operator connected with the problem maps $X_{\alpha}$ into itself and is completely continuous. After that the existence of a solution can be obtained by using either the Schauder fixed point Theorem 3.1 or the Leray-Schauder continuation Theorem 4.1 is devoted similar results for the Carathéodory case. The last result Theorem 6.2 concerns positive solutions. Now (1.1)-(1.2) has a solution $u=u(t) \in C^{2}(0,1)$ if and only if $u \in C[0,1]$ solves the operator equation

$$
u(t)=\int_{0}^{1} G(t, s) f(s, u(s)) d s .
$$


Define the operator $u \mapsto T u$ by

$$
T u(t):=\int_{0}^{1} G(t, s) f(s, u(s)) d s,
$$

where

$$
G(t, s):= \begin{cases}s(1-t) & \text { for } 0 \leq s<t \\ t(1-s) & \text { for } t \leq s \leq 1\end{cases}
$$

is the Green function corresponding to the linear differential operator $-u^{\prime \prime}$ with the boundary value $u(0)=u(1)=0$. Notice that

$$
G(t, s) \leq s(1-s) \quad \text { for all } s, t \in[0,1] .
$$

Remark 2.1. By definition of the Banach space $X_{\alpha}$, we can see that for any $u \in X_{\alpha}$ there is $M>0$ such that for all $t \in(0,1)$, one has

$$
|u(t)| \leq M t^{\alpha}(1-t)^{\alpha} \text {. }
$$

Theorem 2.1. Let $f:(0,1) \times \mathbb{R}^{k} \rightarrow \mathbb{R}^{k}$ be a continuous function. Assume that $f$ satisfies the following condition

$(H)$ : there exist

$$
0<\delta \leq \frac{1}{2}, \quad c>0, \quad \gamma \geq 2, \quad p>\gamma-1
$$

such that for all $t \in(0, \delta) \cup(1-\delta, 1)$, and $|u| \leq \delta$,

$$
|f(t, u)| \leq \frac{c|u|^{p}}{t^{\gamma}(1-t)^{\gamma}}
$$

Then if $\alpha \in(0,1)$ satisfies the inequality

$$
\alpha p+1>\gamma
$$

the operator $T$ maps $X_{\alpha}$ into itself and is completely continuous.

Proof. Let $u \in X_{\alpha}$. By Remark 2.1, there is $M>0$ such that for any $t \in(0,1)$ we have

$$
|u(t)| \leq M t^{\alpha}(1-t)^{\alpha} .
$$

This implies that there exists $0<\delta_{1}<\delta$ such that for $t \in\left(0, \delta_{1}\right) \cup\left(1-\delta_{1}, 1\right)$, one obtains $|u(t)| \leq \delta$. Hence, by (2.2) and (2.3)

$$
\begin{aligned}
|T u(t)| & =\left|\int_{0}^{1} G(t, s) f(s, u(s))\right| d s \\
& \leq\left(\int_{0}^{\delta_{1}}+\int_{\delta_{1}}^{1-\delta_{1}}+\int_{1-\delta_{1}}^{1}\right) s(1-s)|f(s, u(s))| d s \\
& \leq c\|u\|_{\alpha}^{p}\left(\int_{0}^{\delta_{1}}+\int_{1-\delta_{1}}^{1}\right)(s(1-s))^{\alpha p+1-\gamma} d s
\end{aligned}
$$




$$
+\int_{\delta_{1}}^{1-\delta_{1}} s(1-s)|f(s, u(s))| d s .
$$

The first summand is finite by (2.5). Since the interval $\left[\delta_{1}, 1-\delta_{1}\right]$ is closed and the function $u:\left[\delta_{1}, 1-\delta_{1}\right] \rightarrow \mathbb{R}^{k}$ is continuous, hence the subset $u\left(\left[\delta_{1}, 1-\delta_{1}\right]\right)$ is compact in $\mathbb{R}^{k}$.

Put

$$
B:=\left[\delta_{1}, 1-\delta_{1}\right] \times u\left(\left[\delta_{1}, 1-\delta_{1}\right]\right) .
$$

Then $f_{\mid B}$ is a bounded function on the set $B$, i.e., there exists $M_{1}>0$ such that

$$
|f(t, x)| \leq M_{1}
$$

for all $(t, x) \in B$, and in consequence $\int_{\delta_{1}}^{1-\delta_{1}} s(1-s)|f(s, u(s))| d s$ exists. Thus $T$ is well defined. Now we shall prove that $T$ maps $X_{\alpha}$ into itself. First we verify, using the Lebesgue dominated convergence theorem, that:

$$
T u \in C[0,1]
$$

and

$$
\sup _{t \in(0,1)} \frac{|T u(t)|}{t^{\alpha}(1-t)^{\alpha}}<\infty .
$$

In fact, let $\lim _{n \rightarrow \infty} t_{n}=t$. One has

$$
\begin{aligned}
\left|T u\left(t_{n}\right)-T u(t)\right| & \leq\left(\int_{0}^{\delta_{1}}+\int_{\delta_{1}}^{1-\delta_{1}}+\int_{1-\delta_{1}}^{1}\right)\left|G\left(t_{n}, s\right)-G(t, s)\right||f(s, u(s))| d s \\
& \leq\left(\int_{0}^{\delta_{1}}+\int_{1-\delta_{1}}^{1}\right) \varphi_{n}(s)(s(1-s))^{\alpha p-\gamma} d s+\int_{\delta_{1}}^{1-\delta_{1}} \psi_{n}(s) d s .
\end{aligned}
$$

where

$$
\begin{aligned}
& \varphi_{n}(s)=\left.c|| u\right|_{\alpha} ^{p}\left|G\left(t_{n}, s\right)-G(t, s)\right| \\
& \psi_{n}(s)=M\left|G\left(t_{n}, s\right)-G(t, s)\right|,
\end{aligned}
$$

where

$$
M=\sup \{|f(t, u)|:(t, u) \in B\}<\infty,
$$

one obtains

$$
\lim _{n \rightarrow \infty} \psi_{n}(s)=0
$$

(since $G$ is continuous), and

$$
\left|\psi_{n}(s)\right| \leq 2 s(1-s)=: g(s) .
$$

We see that the function $g$ is integrable. Therefore when $n \rightarrow \infty$

$$
\int_{\delta_{1}}^{1-\delta_{1}}\left|G\left(t_{n}, s\right)-G(t, s)\right||f(s, u(s))| d s \rightarrow 0
$$


due to Lebesgue dominated convergence theorem. Now let

$$
\varphi_{n}(s)=c\|u\|_{\alpha}^{p}\left|G\left(t_{n}, s\right)-G(t, s)\right|(s(1-s))^{\alpha p-\gamma} .
$$

By the continuity of the function $G$, we have

$$
\lim _{n \rightarrow \infty} \varphi_{n}(s)=0 \text {, }
$$

and

$$
\varphi_{n}(s) \leq c(s(1-s))^{\alpha p-\gamma+1}
$$

which is an integrable function. Hence

$$
\lim _{n \rightarrow \infty}\left(\int_{0}^{\delta_{1}}+\int_{1-\delta_{1}}^{1}\right) \varphi_{n}(s) d s=0 .
$$

Using (2.9)-(2.10), we have

$$
\lim _{n \rightarrow \infty} T u\left(t_{n}\right)=T u(t) .
$$

Hence $T u \in C[0,1]$. Notice that the map

$$
t \mapsto \frac{1-t}{(t(1-t))^{\alpha}}
$$

is decreasing, and the map

$$
t \mapsto \frac{t}{(t(1-t))^{\alpha}}
$$

is increasing for any $t \in(0,1)$, so one obtains

$$
\frac{1-t}{(t(1-t))^{\alpha}} \leq \frac{1-s}{(s(1-s))^{\alpha}} \quad \text { for } s \leq t,
$$

and

$$
\frac{s}{(s(1-s))^{\alpha}} \leq \frac{t}{(t(1-t))^{\alpha}} \quad \text { for } s \leq t .
$$

Let

$$
H(t, s):=\frac{G(t, s)}{(t(1-t))^{\alpha}} .
$$

By (2.11)-(2.12) we have

$$
H(t, s) \leq(s(1-s))^{1-\alpha} \quad \text { for all } s, t \in[0,1] .
$$

Using (2.11), (2.12) and (2.6), one has

$$
\begin{aligned}
\sup _{t \in(0,1)} \frac{|T u(t)|}{(t(1-t))^{\alpha}} \mid \leq & c|| u \|_{\alpha}^{p}\left(\int_{0}^{\delta_{1}}+\int_{1-\delta_{1}}^{1}\right)(s(1-s))^{\alpha p+1-\alpha-\gamma} d s \\
& +M_{1} \int_{\delta_{1}}^{1-\delta_{1}}(s(1-s))^{1-\alpha} d s<\infty .
\end{aligned}
$$


This shows that for all $u \in X_{\alpha}, T u \in X_{\alpha}$, so that $T: X_{\alpha} \rightarrow X_{\alpha}$. We shall show that $T$ is completely continuous. First we verify the continuity of $T$. In fact, let $\lim _{n \rightarrow \infty} u_{n}=u$ in $X_{\alpha}$. We prove that $T u_{n} \rightarrow T u$ in $X_{\alpha}$. The sequence $u_{n}$ as convergent is bounded, so there exists $M>0$ such that $\left\|u_{n}\right\|_{\alpha} \leq M$ for all $n \in \mathbb{N}$ and $\|u\|_{\alpha} \leq M$. Therefore there is $\delta_{1} \in(0, \delta)$ such that for $t \in\left(0, \delta_{1}\right) \cup\left(1-\delta_{1}, 1\right)$, one has $\left|u_{n}(t)\right| \leq \delta$ for $n \in \mathbb{N}$, and $|u(t)| \leq \delta$. We have

$$
\begin{aligned}
& \left\|T u_{n}-T u\right\|_{\alpha}=\sup _{t \in(0,1)}\left|\frac{T u_{n}(t)-T u(t)}{(t(1-t))^{\alpha}}\right| \\
& \leq\left(\int_{0}^{\delta_{1}}+\int_{\delta_{1}}^{1-\delta_{1}}+\int_{1-\delta_{1}}^{1}\right)(s(1-s))^{1-\alpha}\left|f\left(s, u_{n}(s)\right)-f(s, u(s))\right| d s .
\end{aligned}
$$

Put

$$
\psi_{n}(s):=(s(1-s))^{1-\alpha}\left|f\left(s, u_{n}(s)\right)-f(s, u(s))\right| .
$$

One obtains

$$
\left|\psi_{n}(s)\right| \leq 2(s(1-s))^{1-\alpha}\left|h_{M}(s)\right|,
$$

where

$$
h_{M}(s)= \begin{cases}c M^{p}(s(1-s))^{\alpha p-\gamma}, & s \in\left(0, \delta_{1}\right) \cup\left(1-\delta_{1}, 1\right) \\ M_{1}, & s \in\left[\delta_{1}, 1-\delta_{1}\right] .\end{cases}
$$

Then by the Lebesgue dominated convergence theorem $T u_{n} \rightarrow T u$, as $n \rightarrow$ $\infty$, in $X_{\alpha}$.

We can see that the image $T(D)$ of any bounded set $D \subset X_{\alpha}$ is relatively compact in $X_{\alpha}$, i.e., the family $\{F u: u \in D\}$, where

$$
F u(t):=\frac{T u(t)}{(t(1-t))^{\alpha}},
$$

is uniformly bounded by (2.8) and equicontinuous since the function $H$ has a continuous extension on the product $[0,1] \times[0,1]$, so that $H$ is uniformly continuous, i.e., for a given $\varepsilon>0$ there is $\eta>0$, such that for any $s \in[0,1]$ and $t_{1}, t_{2} \in[0,1]$ if $\left|t_{1}-t_{2}\right|<\eta$ then

$$
\left|H\left(t_{1}, s\right)-H\left(t_{2}, s\right)\right| \leq \max \left(\frac{\varepsilon}{3 c R^{p} \mu}, \frac{\varepsilon}{3 M_{0}}\right),
$$

where

$$
\begin{aligned}
M_{0} & :=\max \left\{|f(t, u(t))|: t \in\left[\delta_{1}, 1-\delta_{1}\right], u \in D\right\}, \\
\mu & :=\int_{0}^{1}(s(1-s))^{\alpha p-\gamma} d s,
\end{aligned}
$$

and $R$ is a radius of the ball containing the set $D$. One has

$$
\left|F u\left(t_{1}\right)-F u\left(t_{2}\right)\right|=\left|\int_{0}^{1}\left(H\left(t_{1}, s\right)-H\left(t_{2}, s\right)\right) f(s, u(s)) d s\right|
$$




$$
\begin{aligned}
& \leq\left(\int_{0}^{\delta_{1}}+\int_{\delta_{1}}^{1-\delta_{1}}+\int_{1-\delta_{1}}^{1}\right)\left|H\left(t_{1}, s\right)-H\left(t_{2}, s\right)\right||f(s, u(s))| d s \\
& \leq c|| u \|_{\alpha}^{p}\left(\int_{0}^{\delta_{1}}+\int_{1-\delta_{1}}^{1}\right)\left|H\left(t_{1}, s\right)-H\left(t_{2}, s\right)\right|(s(1-s))^{\alpha p-\gamma} d s \\
& +\int_{\delta_{1}}^{1-\delta_{1}}\left|H\left(t_{1}, s\right)-H\left(t_{2}, s\right)\right||f(s, u(s))| d s=: J_{1}+J_{2} .
\end{aligned}
$$

We have

$$
\begin{aligned}
J_{1} & \leq c\|u\|_{\alpha}^{p}\left(\int_{0}^{\delta_{1}}+\int_{1-\delta_{1}}^{1}\right) \frac{\varepsilon}{3 c R^{p} \mu}(s(1-s))^{\alpha p-\gamma} d s \\
& \leq \frac{\varepsilon}{3 \mu}\left(\int_{0}^{\delta_{1}}+\int_{1-\delta_{1}}^{1}\right)(s(1-s))^{\alpha p-\gamma} d s \leq 2 \varepsilon / 3 \text { if }\left|t_{1}-t_{2}\right|<\eta,
\end{aligned}
$$

and

$$
J_{2} \leq \int_{\delta_{1}}^{1-\delta_{1}}\left|H\left(t_{1}, s\right)-H\left(t_{2}, s\right)\right||f(s, u(s))| d s \leq \frac{\varepsilon}{3} \text { if }\left|t_{1}-t_{2}\right|<\eta .
$$

Therefore

$$
\left|F u\left(t_{1}\right)-F u\left(t_{2}\right)\right| \leq \varepsilon \quad \text { if }\left|t_{1}-t_{2}\right|<\eta .
$$

This means that the subset $F(D)$ consists of equibounded and equicontinuous functions. Using the Arzèla-Ascoli theorem we can conclude that $T$ is completely continuous.

\section{Application of Schauder theorem}

The following theorem gives a solution to the problem under assumption of the sublinearity of $f$. Unfortunately, $f$ loses his strong singularity at $t=0, t=1$ for large $|u|$.

Theorem 3.1. Assume

(i) there exist

$$
0<\delta \leq \frac{1}{2}, \quad c>0, \quad \gamma \geq 2, \quad p>\gamma-1
$$

such that for any $t \in(0, \delta) \cup(1-\delta, 1), u \in \mathbb{R}^{k}$

$$
|f(t, u)| \leq \frac{c|u|^{p}}{(t(1-t))^{\gamma}}
$$

(ii) there exist

$$
E>0, \quad c_{1}>0, \quad \rho \in(0,1), \quad \nu \leq \frac{\gamma}{p-\rho+1}
$$


such that for all $t \in(0,1)$, and $|u| \geq E$, one has

$$
|f(t, u)| \leq \frac{c_{1}|u|^{\rho}}{(t(1-t))^{\nu}}
$$

Let $\alpha \in(0,1)$ satisfy the condition $(2.5)$. Set

$$
\begin{gathered}
\omega:=\alpha p+1-\alpha-\gamma, \quad \xi:=\alpha \rho+1-\alpha-\nu \\
\lambda_{2}:=\int_{\delta}^{1-\delta}(s(1-s))^{\xi} d s \quad \text { and } \quad \lambda_{1}:=\int_{\delta}^{1-\delta}(s(1-s))^{1-\alpha} d s .
\end{gathered}
$$

Let

$$
M:=\sup \{|f(t, u)|:|u| \leq E ; \delta \leq t \leq 1-\delta\} .
$$

If there is a positive number $R$ such that

$$
R \geq c R^{p}\left(\int_{0}^{\delta}+\int_{1-\delta}^{1}\right)(s(1-s))^{\omega} d s+\max \left(M \lambda_{1}, c_{1} \lambda_{2} R^{\rho}\right) .
$$

Then the problem (1.1)-(1.2) has a solution in $X_{\alpha}$ with the norm $\|u\|_{\alpha} \leq R$.

Proof. Let

$$
\bar{B}(0, R):=\left\{u \in X_{\alpha}:\|u\|_{\alpha} \leq R\right\}
$$

be a closed ball in $X_{\alpha}$ centered at 0 with radius defined in (3.3). We shall prove that $T$ maps this ball into itself, i.e., $T(\bar{B}(0, R)) \subset \bar{B}(0, R)$. In fact, let $u \in \bar{B}(0, R)$, one obtains

$$
\begin{aligned}
\sup _{t \in(0,1)}\left|\frac{T u(t)}{(t(1-t))^{\alpha}}\right| & \leq\left(\int_{0}^{\delta}+\int_{\delta}^{1-\delta}+\int_{1-\delta}^{1}\right)(s(1-s))^{1-\alpha}|f(s, u(s))| d s \\
& \leq c R^{p}\left(\int_{0}^{\delta}+\int_{1-\delta}^{1}\right)(s(1-s))^{\omega} d s \\
& +\int_{\delta}^{1-\delta}(s(1-s))^{1-\alpha} \max \left(M, c_{1}\|u\|_{\alpha}^{\rho}(s(1-s))^{\alpha \rho-\nu}\right) d s .
\end{aligned}
$$

So for $\|u\|_{\alpha} \leq R$, using (3.3), one has $\|T u\|_{\alpha} \leq R$. Hence $T$ has a fixed point due to Schauder Fixed Point Theorem.

Remark 3.1. First assumption of the above theorem is slightly stronger than condition $(H)$ which guarantees the complete continuity of $T$. 


\section{Example 3.1.}

$$
f(t, u)= \begin{cases}\frac{u^{4}}{(t(1-t))^{3}} & \text { for }|u| \leq 1 \\ \frac{|u|^{1 / 2}}{(t(1-t))^{1 / 2}} & \text { for }|u| \geq 4 \\ {\left[\frac{2}{3(t(1-t))^{1 / 2}}-\frac{1}{3(t(1-t))^{3}}\right]|u|+\frac{4}{3(t(1-t))^{3}}-\frac{2}{3(t(1-t))^{1 / 2}}} & \text { for }|u| \in(1,4) .\end{cases}
$$

Let $\delta=1 / 2$ and $p=4, \gamma=3$ when $t$ is near the end points $t=0, t=1$ of the interval $[0,1]$, and $u \in \mathbb{R}$ with $|u|$-small, and

$$
\rho=\nu=\frac{1}{2}
$$

when $t$ is everywhere on $(0,1)$ with $|u| \geq 4=: E$ and let $\alpha \in(1 / 2,5 / 7)$, then by assumption, one has

$$
\frac{1}{2}=\nu \leq \frac{\gamma}{p-\rho+1}=\frac{2}{3} .
$$

Let now

$$
\alpha=\frac{5}{7}, \quad p=4, \quad \gamma=3, \quad \rho=\nu=\frac{1}{2}, \quad \delta=\frac{1}{2}, \quad \text { and } \quad c=c_{1}=1,
$$

then for these values the condition $\alpha p+1>\gamma$ is satisfied and

$$
\begin{aligned}
& \omega=1-\alpha+\alpha p-\gamma=\frac{1}{7}, \quad \xi=1-\alpha+\alpha \rho-\nu=\frac{1}{7}, \\
& \lambda_{1}=\int_{\delta}^{1-\delta}(s(1-s))^{1-\alpha} d s=0, \quad \lambda_{2}=\int_{\delta}^{1-\delta}(s(1-s))^{\xi} d s=0, \\
& \left(\int_{0}^{\delta}+\int_{1-\delta}^{1}\right)(s(1-s))^{\omega} d s=\int_{0}^{1}(s(1-s))^{\frac{1}{7}} d s=\frac{1}{294} .
\end{aligned}
$$

It is easy to verify that there exists $R>0$ which satisfies

$$
\frac{1}{294} R^{4} \leq R
$$

Remark 3.2. The integral is computed by MAPLE 6 . 


\section{Application of the topological degree}

We shall use the following theorem

Theorem 4.1 ([6, Lemma 2.5.1]). Let $\Omega$ be a bounded open set in a real Banach space $E, 0 \in \Omega$ and $A: \bar{\Omega} \rightarrow E$ be completely continuous. Suppose $A u \neq \mu u$, for all $u \in \partial \Omega, \mu \geq 1$. Then the operator $A$ has a fixed point in $\Omega$.

Theorem 4.2. Suppose condition $(H)$ holds and $\alpha$ satisfies (2.5). Assume that there exists $M>0$ such that for any $|u|>M(t(1-t))^{\alpha}$, one has

$$
(f(t, u), u) \leq 0 \quad \text { for all } t \in(0,1) .
$$

Then the Dirichlet problem (1.1)-(1.2) has a solution in $X_{\alpha}$.

Proof. First let us assume that inequality (4.1) is sharp. Let $B\left(0, M_{0}\right)$ be a ball in $X_{\alpha}$ centered at 0 with radius $M_{0}$, where $M_{0}=M+1$. We shall prove that the BVP

$$
u^{\prime \prime}=-\lambda f(t, u), \quad u(0)=u(1)=0, \quad \text { for } \lambda \in(0,1],
$$

has no solutions on $\partial B\left(0, M_{0}\right)$. Suppose on the contrary that there exist $\varphi$ and $\lambda>0$ satisfying (4.2) such that $\|\varphi\|_{\alpha}=M_{0}$. Put

$$
\psi(t):=\frac{\varphi(t)}{(t(1-t))^{\alpha}} .
$$

Since $\varphi$ satisfies (4.2), then it is of the form

$$
\varphi(t)=\lambda \int_{0}^{1} G(t, s) f(s, \varphi(s)) d s .
$$

We can see that

$$
\varphi^{\prime}(t)=-\lambda \int_{0}^{t} s f(s, \varphi(s)) d s+\lambda \int_{t}^{1}(1-s) f(s, \varphi(s)) d s
$$

So by condition $(H)$, one has $\varphi^{\prime}$ is a bounded function and then, due to the l'Hospital theorem

$$
\lim _{t \rightarrow 0,1} \psi(t) \rightarrow 0
$$

Since the function $\psi$ is continuous, hence there is $t_{0} \in(0,1)$ such that

$$
\left|\frac{\varphi\left(t_{0}\right)}{\left(t_{0}\left(1-t_{0}\right)\right)^{\alpha}}\right|=M_{0}
$$

Then $\left|\psi\left(t_{0}\right)\right|=M_{0}$, and one has

$$
0=\frac{d}{d t}|\psi(t)|_{t=t_{0}}^{2}=\frac{d}{d t}(\psi(t), \psi(t))_{t=t_{0}}=2\left(\psi^{\prime}\left(t_{0}\right), \psi\left(t_{0}\right)\right) .
$$


Using (4.1)

$$
0 \geq \frac{d^{2}}{d t^{2}}|\psi(t)|_{t=t_{0}}^{2}=2\left(\psi^{\prime \prime}\left(t_{0}\right), \psi\left(t_{0}\right)\right)>0
$$

a contradiction. Any solution of (4.2) is a zero of the operator $(1 / \lambda) I-T$. Hence, by Theorem 4.1 with $A=T, \mu=1 / \lambda$, the equation $u-T u=0$ has a solution $u \in \Omega$.

Now, pass to the general case: inequality (4.1) is as in the statement of the theorem. Perturbing the right-hand side of the differential equation by $-(1 / n) u$, where $n \in \mathbb{N}$, we have a solution $u_{n}$ by the first part of the proof. It is easily seen that the sequence $\left(u_{n}\right)_{n}$ satisfies the assumptions of the Arzéla-Ascoli theorem. Thus it has a uniformly convergent subsequence $u_{n_{m}} \rightarrow u$. The limit is a solution of the main problem.

Example 4.1. Let

$$
f(t, u)=\frac{-u^{2 n+1}}{(t(1-t))^{\gamma}}+h(t)
$$

where $h \in X_{\alpha}, t \in(0,1), u \in \mathbb{R}^{+}, 2 n+1>\gamma-1$, and $1>\alpha>$ $(\gamma-1) /(2 n+1)$. If $h \in X_{\alpha}$ we have

$$
|h(t)| \leq M(t(1-t))^{\alpha}
$$

and for $|u| \geq M(t(1-t))^{\alpha}$, one has

$$
u f(t, u) \leq 0 \text {. }
$$

It is obvious, since

for such $u$.

$$
|u h(t)| \leq \frac{|u|^{2 n+2}}{(t(1-t))^{\gamma}}
$$

\section{The Carathéodory conditions}

Consider the Dirichlet problem (1.1)-(1.2) with $f:(0,1) \times \mathbb{R}^{k} \rightarrow \mathbb{R}^{k}$ satisfying the Carathéodory conditions, i.e., $f(\cdot, u): t \mapsto f(t, u)$ is measurable on $(0,1)$ for each $u \in \mathbb{R}^{k}$ and $f(t, \cdot): u \mapsto f(t, u)$ is continuous on $\mathbb{R}^{k}$ for almost all $t \in(0,1)$. Let

$$
L_{\{1-\alpha\}}:=\left\{h: \int_{0}^{1}(s(1-s))^{1-\alpha}|h(s)| d s<\infty\right\}, \quad \alpha \in(0,1)
$$

be the $L^{1}$-space for the measure $\mu$ on $[0,1]$ defined by the formula

$$
\mu(A)=\int_{A}(s(1-s))^{1-\alpha} d s .
$$


Theorem 5.1. Suppose that $f$ satisfies the Carathéodory conditions, and for any $M>0$ there is $h_{M} \in L_{\{1-\alpha\}}$, such that for any $|u| \leq M(t(1-t))^{\alpha}$ we obtain

$$
|f(t, u)| \leq h_{M}(t)
$$

for a. e. $t \in(0,1)$. Then the operator $T$ is completely continuous from $X_{\alpha}$ into $X_{\alpha}$, where $T$ is of the form (2.2).

Proof. By assumption and by the fact that

$$
t(1-t) \leq(t(1-t))^{1-\alpha},
$$

for $\alpha \in(0,1)$, and for any $t \in[0,1]$, one has

$$
|G(t, s) f(s, \varphi(s))| \leq s(1-s)\left|h_{M}(s)\right|
$$

for $\|\varphi\|_{\alpha} \leq M$. This implies that

$$
\int_{0}^{1} s(1-s)\left|h_{M}(s)\right| d s<\infty
$$

and in consequence $(2.2)$ exists. We prove that the operator $T$ maps $X_{\alpha}$ into itself. Let

$$
F u(t):=\frac{T u(t)}{(t(1-t))^{\alpha}} .
$$

So the operator $F$ is of the form:

$$
F u(t):=\frac{T u(t)}{(t(1-t))^{\alpha}}=\int_{0}^{1} H(t, s) f(s, u(s)) d s .
$$

Let $\varphi \in X_{\alpha}$ such that $\|\varphi\|_{\alpha} \leq M$, so there exists $h_{M} \in L_{\{1-\alpha\}}$, and

$$
\begin{aligned}
\left|F \varphi(t)-F \varphi\left(t_{0}\right)\right| & \leq \int_{0}^{1}\left|H(t, s)-H\left(t_{0}, s\right)\right||f(s, \varphi(s))| d s \\
& \leq \int_{0}^{1}\left|H(t, s)-H\left(t_{0}, s\right)\right|\left|h_{M}(s)\right| d s .
\end{aligned}
$$

Let

$$
\psi_{t}(s):=\left|H(t, s)-H\left(t_{0}, s\right)\right|\left|h_{M}(s)\right| .
$$

Since the function $H$ is uniformly continuous on the product $[0,1] \times[0,1]$, then

$$
\lim _{t \rightarrow t_{0}}\left|H(t, s)-H\left(t_{0}, s\right)\right|\left|h_{M}(s)\right|=0
$$

uniformly with respect to $t_{0} \in[0,1]$, and

$$
\left|\psi_{t}(s)\right| \leq 2(s(1-s))^{1-\alpha}\left|h_{M}(s)\right|=: g(s) .
$$

Hence, using the Lebesgue dominated convergence theorem again, one has

$$
\lim _{t \rightarrow t_{0}} \int_{0}^{1}\left|H(t, s)-H\left(t_{0}, s\right)\right|\left|h_{M}(s)\right| d s=0 .
$$


So

$$
\lim _{t \rightarrow t_{0}}\left|F \varphi(t)-F \varphi\left(t_{0}\right)\right|=0
$$

for any $t_{0} \in(0,1)$. This means that the function $F \varphi$ is continuous on $[0,1]$, so that $F \varphi$ is bounded and in consequence $T \varphi \in X_{\alpha}$ for any $\varphi \in X_{\alpha}$. Now we shall prove that the subset $T(\bar{B}(0, M))$ is relatively compact in $X_{\alpha}$, i.e., the subset $F(\bar{B}(0, M))$ consists of equibounded and equicontinuous functions. By (5.2), for any $\varepsilon>0$ there is $\delta>0$ such that if $\left|t-t_{0}\right|<\delta$ implies

$$
\int_{0}^{1}\left|H(t, s)-H\left(t_{0}, s\right)\right|\left|h_{M}(s)\right| d s \leq \varepsilon,
$$

then for any function $\|\varphi\|_{\alpha} \leq M$ and $\left|t-t_{0}\right|<\delta$

$$
\left|F \varphi(t)-F \varphi\left(t_{0}\right)\right| \leq \int_{0}^{1}\left|H(t, s)-H\left(t_{0}, s\right)\right|\left|h_{M}(s)\right| d s \leq \varepsilon
$$

i.e., the family $\left\{F \varphi:\|\varphi\|_{\alpha} \leq M\right\}$ is equicontinuous. Moreover for $\varphi \in X_{\alpha}$ and $\|\varphi\|_{\alpha} \leq M$ there exists $h_{M} \in L_{\{1-\alpha\}}$ such that

$$
\begin{aligned}
\sup _{t \in(0,1)}|F \varphi(t)| & \leq \sup _{t \in(0,1)} \int_{0}^{1} H(t, s) \mid f(s, \varphi(s) \mid d s \\
& \leq \int_{0}^{1}(s(1-s))^{1-\alpha}\left|h_{M}(s)\right| d s=: N<+\infty .
\end{aligned}
$$

Then the family $\left\{F \varphi:\|\varphi\|_{\alpha} \leq M\right\}$ is equibounded. By Arzéla-Ascoli theorem the operator $T$ is compact in $X_{\alpha}$. Now we shall prove that $T$ is continuous. In fact, let $\varphi_{n}$ be a sequence of elements in $X_{\alpha}$, converging to some function $\varphi$ of $X_{\alpha}$, i.e.,

$$
\left\|\varphi_{n}-\varphi\right\|_{\alpha} \rightarrow 0
$$

when $n \rightarrow \infty$. There is $M>0$ such that $\left\|\varphi_{n}\right\|_{\alpha} \leq M$ for all $n \in \mathbb{N}$ and $\|\varphi\|_{\alpha} \leq M$. By assumption on $f$, one has

$$
\lim _{n \rightarrow \infty} f\left(t, \varphi_{n}(t)\right)=f(t, \varphi(t))
$$

for almost all $t$. Let $\varepsilon>0$. Since the integral

$$
\int_{0}^{1}(s(1-s))^{1-\alpha}\left|h_{M}(s)\right| d s
$$

exists then there is $\delta>0$ such that for $J \subset I$, where $I=[0,1]$, and $\mu(J)<\delta$

$$
\int_{J}(s(1-s))^{1-\alpha}\left|h_{M}(s)\right| d s \leq \frac{\varepsilon}{4 \mu(I)} .
$$

Using the Egoroff theorem there is $J_{1} \subset I$ such that for $\mu\left(J_{1}\right) \leq \delta$

$$
\lim _{n \rightarrow \infty} f\left(t, \varphi_{n}(t)\right)=f(t, \varphi(t))
$$


uniformly on $I-J_{1}$, so there exists $n_{0} \in \mathbb{N}$, and for $n \geq n_{0}$

$$
(s(1-s))\left|f\left(s, \varphi_{n}(s)\right)-f(s, \varphi(s))\right| \leq \frac{\varepsilon}{2 \mu(I)}
$$

for $s \in I-J_{1}$. Therefore for all $n \geq n_{0}$

$$
\begin{aligned}
\sup _{t \in(0,1)}\left|F \varphi_{n}(t)-F \varphi(t)\right| & \leq\left(\int_{I-J_{1}}+\int_{J_{1}}\right) H(t, s)\left|f\left(s, \varphi_{n}(s)\right)-f(s, \varphi(s))\right| d s \\
& \leq \frac{\varepsilon}{2 \mu(I)} \int_{I-J_{1}} \mu d s+2 \int_{J_{1}}(s(1-s))^{1-\alpha}\left|h_{M}(s)\right| d s \\
& \leq \frac{\varepsilon}{2}+\frac{\varepsilon}{2}=\varepsilon .
\end{aligned}
$$

Then

$$
\left\|T \varphi_{n}-T \varphi\right\|_{\alpha} \leq \varepsilon
$$

This means that the operator $T$ is continuous.

Now to prove that the problem (1.1)-(1.2) has a solution, we can repeat Theorem 4.2 and the application of the Theorem 3.1 for Carathéodory functions.

\section{Positive solutions}

Now we look for a positive solution to problem (1.1)-(1.2) in dimension $k=1$, for simplicity. Function $f:(0,1) \times \mathbb{R}_{+} \rightarrow \mathbb{R}_{+}\left(\mathbb{R}_{+}=[0, \infty)\right)$ is supposed to be continuous or to satisfy the Carathéodory conditions. Let $E$ be a real Banach space and $P$ denote a cone in $E$, i.e. $P \subset E$ is a nonempty closed convex set such that

$$
\begin{aligned}
& u \in P, \quad \lambda \geq 0 \quad \Rightarrow \quad \lambda u \in P \\
& u \in P \cap(-P) \quad \Rightarrow \quad u=0 .
\end{aligned}
$$

This cone defines a partial order in $E$ :

$$
u \leq v \quad \Leftrightarrow \quad v-u \in P
$$

and one can set

$$
[u, v]:=\{w \in E: \quad u \leq w \leq v\} .
$$

Any operator defined on a subset of $E$ is called increasing if

$$
u \leq v \quad \Rightarrow \quad T u \leq T v .
$$

If $u \leq v$ and $u \neq v$, we write $u<v$.

We shall use the following theorem on fixed points for increasing operators. 
Theorem 6.1 ([6, Theorem 2.1.3]). Let $E$ be a real Banach space, let $u_{0}, v_{0} \in E, u_{0}<v_{0}$ and $T:\left[u_{0}, v_{0}\right] \rightarrow E$ be an increasing operator such that $u_{0} \leq T u_{0}, T v_{0} \leq v_{0}$. Suppose that $T\left(\left[u_{0}, v_{0}\right]\right)$ is a relatively compact subset of $E$. Then $T$ has at least one fixed point in $\left[u_{0}, v_{0}\right]$.

Now let $P_{\alpha}:=\left\{u \in X_{\alpha}: u(t) \geq 0, t \in[0,1]\right\}$ be a cone in the real Banach space $X_{\alpha}$. Similarly as in Section 2, we can prove that under condition $(H)$, (Theorem 2.1), the operator $T$ maps $X_{\alpha}$ into itself, and $T: X_{\alpha} \rightarrow X_{\alpha}$ is completely continuous.

Theorem 6.2. Let $f$ be a non-negative continuous function satisfying the condition $(H)$, and there exist $r_{0}>0, c_{0}>0, \beta>-2$ such that for any $t \in(0,1)$, one has:

$$
\begin{aligned}
f\left(t, r_{0} t(1-t)\right) & \geq c_{0}(t(1-t))^{\beta}, \\
\lambda c_{0} & \geq r_{0},
\end{aligned}
$$

where

$$
\lambda=\int_{0}^{1}(s(1-s))^{1+\beta} d s .
$$

Suppose that there exists $\alpha \leq 1 / 2$ satisfying the condition

$$
\alpha p+1>\gamma
$$

and

$$
\begin{gathered}
\lim _{u \rightarrow \infty} \sup _{t \in(0,1)} \frac{f(t, u)}{u}(t(1-t))^{\alpha}=0, \\
f(t, \cdot) \text { is non-decreasing on } \mathbb{R}^{+} \text {for } 0<t<1 .
\end{gathered}
$$

Then the operator $T$ has a fixed point in $P_{\alpha}$.

Proof. Define the operator $u \mapsto T u$ as in (2.2). Put

$$
u_{0}(t)=r_{0} t(1-t) .
$$

Since $t(1-t) \leq(t(1-t))^{\alpha}$ then $u_{0}(t) \leq r_{0}(t(1-t))^{\alpha}$ and using (6.1), one has

$$
\begin{aligned}
T u_{0}(t) & =\int_{0}^{1} G(t, s) f\left(s, u_{0}(s)\right) d s \\
& \geq c_{0} r_{0}\left((1-t) \int_{0}^{t} s^{1+\beta}(1-s)^{\beta} d s+t \int_{t}^{1} s^{\beta}(1-s)^{\beta+1} d s\right) \\
& \geq c_{0} r_{0} t(1-t) \int_{0}^{1}(s(1-s))^{1+\beta} d s
\end{aligned}
$$


By (6.2)-(6.3), one has

$$
c_{0} r_{0} t(1-t) \int_{0}^{1}(s(1-s))^{1+\beta} d s \geq r_{0} t(1-t)=u_{0}(t) .
$$

Therefore $T u_{0} \geq u_{0}$. Using (6.4) one obtains: there exists $R>r_{0}$ such that

$$
\frac{f(t, R)}{R} \leq \frac{1}{(t(1-t))^{\alpha}},
$$

for any $t \in(0,1)$. Let $v_{0}(t)=R(t(1-t))^{1-\alpha}$. We observe that $v_{0} \in X_{\alpha}$, (since $\alpha \leq 1 / 2), v_{0}(t)<R$, for all $t \in[0,1]$, and $\alpha \in(0,1), u_{0}(t)<v_{0}(t)$, for any $t \in[0,1]$. By (6.7), (2.11) and (2.12) we have

$$
\begin{aligned}
T v_{0}(t) & =\int_{0}^{1} G(t, s) f\left(s, v_{0}(s)\right) d s \leq \int_{0}^{1} G(t, s) f(s, R) d s \\
& \leq R \int_{0}^{t}(1-t) \frac{s d s}{(s(1-s))^{\alpha}}+R \int_{t}^{1} t \frac{(1-s) d s}{(s(1-s))^{\alpha}} \\
& \leq R \int_{0}^{t}(1-t) \frac{t d s}{(t(1-t))^{\alpha}}+R \int_{t}^{1} t \frac{(1-t) d s}{(t(1-t))^{\alpha}} \\
& =R(t(1-t))^{1-\alpha} \int_{0}^{1} d s=v_{0}(t) .
\end{aligned}
$$

So for $0 \leq t \leq 1$ we have

$$
T v_{0}(t) \leq v_{0}(t)
$$

We can apply Theorem 6.1. Therefore the operator $T$ has one positive solution.

Example 6.1. Let $p>\gamma-1$. The following function satisfies all assumptions of the last theorem.

$$
f(t, u):= \begin{cases}\frac{u^{p}}{t^{\gamma}(1-t)^{\gamma}} & \text { for } 0 \leq u \leq t(1-t), \\ t^{p-\gamma}(1-t)^{p-\gamma} & \text { for } u>t(1-t) .\end{cases}
$$

For $0 \leq u \leq v \leq t(1-t)$

$$
f(t, u)-f(t, v)=\frac{u^{p}-v^{p}}{t^{\gamma}(1-t)^{\gamma}} \leq 0
$$

(since $p>\gamma-1 \geq 1)$. For $u \leq t(1-t)$, and $v>t(1-t)$, one has

$$
\begin{aligned}
f(t, u)-f(t, v) & =\frac{u^{p}}{t^{\gamma}(1-t)^{\gamma}}-t^{p-\gamma}(1-t)^{p-\gamma} \\
& \leq t^{p-\gamma}(1-t)^{p-\gamma}-t^{p-\gamma}(1-t)^{p-\gamma}=0 .
\end{aligned}
$$

Now for $t(1-t)<u \leq v$, we have

$$
f(t, u)-f(t, v)=0 .
$$


Hence the function $f(t, \cdot)$ is increasing for any $t \in(0,1)$. We can see that

$$
\lim _{u \rightarrow \infty} \frac{f(t, u)}{u} t^{\alpha}(1-t)^{\alpha}=0 .
$$

If $u>r_{0} t(1-t)$ and $r_{0} \geq 1$, then by (6.5) we note that

$$
f(t, u) \geq c_{0} t^{\beta}(1-t)^{\beta}
$$

and from the assumption, one has

$$
f(t, u)=t^{p-\gamma}(1-t)^{p-\gamma} .
$$

So the inequality

$$
t^{p-\gamma}(1-t)^{p-\gamma} \geq c_{0} t^{\beta}(1-t)^{\beta}
$$

must be satisfied and, in consequence, $p-\gamma \leq \beta$.

Remark 6.1. All examples in this paper are not natural and complicated but they demonstrate the fact that such examples exist.

Remark 6.2. We have tried to apply the Krasnoselskii Fixed Point Theorem for cone-expansion maps but it seems that it is impossible in our situation.

\section{References}

[1] Avery, R. I., Davis, J. M., Henderson, J., Three symmetric positive solutions for Lidstone problems by a generalization of the Leggett-Williams theorem, Electron. J. Differential Equations 40 (2000), 1-15.

[2] Dunninger, D. R., Kurtz, J. C., A priori bounds and existence of positive solutions for singular nonlinear boundary value problems, SIAM J. Math. Anal. 3 (1986), 595-609.

[3] Eloe, P. W., Henderson, J., Positive solutions for $(n-1,1)$ conjugate boundary value problems, Nonlinear Anal. 10 (1997), 1669-1680.

[4] Gatica, J. A., Oliker, V., Waltman, P., Singular nonlinear boundary value problems for second-order ordinary differential equations, J. Differential Equations 79 (1989), $62-78$.

[5] Gatica, J. A., Oliker, V., Waltman, P., Iterative procedures for nonlinear second order boundary value problems, Ann. Mat. Pura Appl. 4 (1990), 1-25.

[6] Guo, D., Lakshmikantham, V., Nonlinear Problems in Abstract Cones, Harcourt Brace Jovanovich, Publishers, Boston-San Diego-New York-Berkeley-LondonSydney-Tokyo-Toronto, 1988.

[7] Habets, P, Zanolin, F., Upper and lower solutions for a generalized Emden-Fowler equation, J. Math. Anal. Appl. 181 (1994), 684-700.

[8] Jiang, D., Gao, W., Upper and lower solution method and a singular BVP for the 1-dimensional p-Laplacian, J. Math. Anal. Appl. 252 (2000), 631-648.

[9] Lloyd, N. G., Degree Theory, Cambridge Tracts in Mathematics 73, Cambridge Univ. Press, Cambridge, 1978.

[10] Ma, R., Positive solutions of a nonlinear three-point boundary-value problem, Electron. J. Differential Equations 34 (1998), 1-8. 
[11] Martin, R. H., jr., Nonlinear Operators and Differential Equations in Banach Spaces, John Wiley \& Sons, New York-London-Sydney-Toronto, 1976.

[12] Przeradzki, B., Stańczy, R., Positive solutions for sublinear elliptic equations, Colloq. Math. 92 (2002), 141-151.

[13] O'Regan, D., Singular Dirichlet boundary value problems - superlinear and nonresonant case, Nonlinear Anal. 2 (1997), 221-245.

[14] Taliaferro, S. D., A nonlinear singular boundary value problem, Nonlinear Anal. 6 (1979), 897-904.

[15] Liu, Z., Li, F., Multiple positive solutions of nonlinear two-point boundary value problems, J. Math. Anal. Appl. 203 (1996), 610-625.

\author{
Habib Sebai \\ FACULTY OF MATHEMATICS \\ UNIVERSITY OF ŁÓDŹ \\ BANACHA 22 \\ 90-238 ŁóDŹ, POLAND \\ E-MAIL: HABIBSEBAI@OP.PL
}

